\section{Essential Oil Composition and Yield of Anise from Different Distillation Times}

\author{
Valtcho D. Zheljazkov ${ }^{1}$ \\ University of Wyoming, Sheridan Research and Extension Center, 663 \\ Wyarno Road, Sheridan, WY 82801
}

Tess Astatkie

Dalhousie University, Faculty of Agriculture, 50 Pictou Road, P.O. Box 550, Truro, Nova Scotia B2N 5E3, Canada

Barry O'Brocki

Citrus and Allied, 4620 Mercedes Drive, Belcamp, MD 21017

\section{Ekaterina Jeliazkova}

University of Wyoming, Sheridan Research and Extension Center, 663 Wyarno Road, Sheridan, WY 82801

Additional index words. anethole, linalool, methyl chavicol, Pimpinella anisum, steam distillation

\begin{abstract}
Anise (Pimpinella anisum L.) is a spice, an essential oil crop, and a medicinal plant with a long history of use. Anise seed oil is extracted from anise seed through steam distillation. There is no experimentally established optimal time for distillation of anise seed. We hypothesized that the distillation time (DT) can be customized for optimum yield and composition of anise essential oil. In this study, we determined the effect of nine steam DTs $(5,15,30,60,120,180,240,360$, and 480 minutes) on essential oil yield and essential oil composition of anise seed. We developed regression models to predict essential oil yield, the concentration of individual constituents, and the yield of these constituents as a function of DT. Highest essential oil yield $(2.0 \mathrm{~g} / 100 \mathrm{~g}$ seed, $2 \%)$ was obtained at 360-minute DT. The concentration of transanethole, the major anise oil constituent, varied from $93.5 \%$ to $96.2 \%$ (as a percent of the total oil) and generally was high at 15- to 60-minute DT and low at 240- to 480-minute DT. However, the yield of transanethole (calculated from the essential oil yield and the concentration of transanethole in the oil) increased with increasing DT to reach maximum at 360-minute DT. The concentration of the other oil constituents varied significantly depending on the DT, and some of them were higher at the shorter DT than at the longer DT. However, the yields of these constituents were highest at longer DT (either 360 or 480 minutes). DT can be used to obtain anise essential oil with different composition that would benefit the essential oil industry. This study demonstrated the need for providing DT in reports where anise seed essential oil yield and composition are discussed. This article can also be used as a reference point for comparing studies in which different DTs were used to extract essential oil from anise seed.
\end{abstract}

Anise (Pimpinella anisum L.) is one of the most widely used plants in the world; anise seed is used as a spice (Leela and Vipin, 2008), and anise seed essential oil has a number of applications as an aromatic agent in the food and liquor industry (Leela and Vipin, 2008; Stojanov, 1973; Tonutti and Liddle, 2010). Commercial production of anise seed and anise essential oil is concentrated in countries in south Asia, Europe, North Africa, and also in Russia. Anise has been used as a medicinal plant since ancient times (Leela and Vipin, 2008). Many folk medicinal

Received for publication 7 June 2013. Accepted for publication 25 Sept. 2013.

This research was supported by start-up funds from the University of Wyoming awarded to Dr. V.D. Zheljazkov.

${ }^{1}$ To whom reprint requests should be addressed; e-mailvjeliazk@uwyo.edu, valtcho.pubs@gmail.com. anis, and many other alcoholic and nonalcoholic beverages (Tonutti and Liddle, 2010). Anise essential oil has also antimicrobial and other medicinal properties (Gende et al., 2009; Leela and Vipin, 2008) and has been explored as a food preservative and for decreasing aflatoxin B1 in maize grain (Bluma and Etcheverry, 2008).

Anise seed essential oil content and composition vary depending on the environment and the genotype (Leela and Vipin, 2008). Steam distillation has been traditionally used for extracting essential oil from anise seed. Steam DT has been demonstrated to alter essential oil yield and composition of other crops such as peppermint (Mentha $\times$ piperita L.), lemongrass (Cymbopogon flexuosus Steud.), and palmarosa (Cymbopogon martini Roxb.) (Cannon et al., 2013), pine (Pinus ponderosa Dougl. ex Laws) (Zheljazkov et al., 2012a), Japanese cornmint (Mentha canadensis L.) (Zheljazkov and Astatkie, 2012), oregano (Origanum vulgare L.) (Zheljazkov et al., 2012b), female and male Rocky Mountain juniper (Juniperus scopulorum Sarg.) (Zheljazkov et al., 2012c, 2013). However, there are no reports on the effect of DT on anise seed essential oil yield and composition. We hypothesized that DT can alter anise seed essential oil yield and composition, and hence, DT can be customized to obtain essential oil with a desired profile.

\section{Materials and Methods}

Steam distillation and distillation times. All steam DTs were conducted in 2-L steam distillation units (Hearthmagic, Rancho Santa Fe, CA) as described previously for extraction of basil essential oil (Zheljazkov et al., 2008) and for the distillation of Juniperus virginiana L. (Gawde et al., 2009).

Certified organic bulk anise seed was purchased from Starwest Botanicals (Rancho Cordova, CA). The country of origin for the organic anise seed used in this study was Egypt. The DT experiments were conducted at the University of Wyoming Sheridan Research and Extension Center in 2012. The sample size was $500 \mathrm{~g}$ of anise seed; all samples were generated randomly from the bulk seed lot. All DTs were performed in three replicates. Nine steam DTs were studied: $5 \mathrm{~min}, 15 \mathrm{~min}, 30 \mathrm{~min}, 60 \mathrm{~min}, 120 \mathrm{~min}$, $180 \mathrm{~min}, 240 \mathrm{~min}, 360 \mathrm{~min}$, and $480 \mathrm{~min}$. We have chosen the DTs based on our preliminary studies, indicating that sufficient amount (for handling and analyses) of essential oil can be obtained at 5-min DT, on literature data, and on the commonly used DT by the essential oil industry extracting anise oil (Dr. Nedko Nedkov, personal communication). The individual DTs were measured from the beginning of the distillation, considered the moment the first drop of essential oil was deposited. At the end of each individual DT, the power was turned off and the Florentine vessel (the separator) removed from the apparatus. The extracted essential oils were separated, measured on an analytical 
scale, and placed in a freezer at $-5{ }^{\circ} \mathrm{C}$ until they were analyzed. The essential oil yield (content) was calculated as grams of oil per $100 \mathrm{~g}$ weight of anise seed. The water content of the seed was $8.9 \%$.

Gas chromatography analysis of essential oil. The anise seed essential oil samples (all samples in three replicates) were analyzed on a gas chromatograph (GC; Hewlett Packard Model 6890; Hewlett-Packard, Palo Alto, CA). The carrier gas was helium at flow rate of $40 \mathrm{~cm} \cdot \mathrm{sec}^{-1}, 11.7 \mathrm{psi}\left(60^{\circ} \mathrm{C}\right)$, and $2.5 \mathrm{~mL} \cdot \mathrm{min}^{-1}$ constant flow rate. The injection was split $60: 1,0.5 \mu \mathrm{L}$, and the injector temperature was $220{ }^{\circ} \mathrm{C}$. The $\mathrm{GC}$ oven temperature program was as follows: $60{ }^{\circ} \mathrm{C}$ for $1 \mathrm{~min}$ and $10{ }^{\circ} \mathrm{C} / \mathrm{min}$ to $250{ }^{\circ} \mathrm{C}$. The column was HP-INNOWAX (crosslinked polyethylene glycol; $30 \mathrm{~m} \times 0.32 \mathrm{~mm} \times$ $0.5 \mu \mathrm{m})$, and the flame ionization detector temperature was $275^{\circ} \mathrm{C}$. Individual constituents of the anise essential oil are expressed as percentage of the total oil. The identification of individual constituent peaks was done with the use of internal standards by retention time and by mass spectroscopy.

Statistical analyses. The effect of DT on essential oil content and on the concentration and yield of linalool, methyl chavicol, para-anis-aldehyde, transanethole, gammahimachalene, transpseudoisoeugenyl-2-methyl, and epoxy-pseudoisoeugenyl-2-methyl was determined using a one-way analysis of variance. For each response, the validity of model assumptions was verified by examining the residuals as described in Montgomery (2013). Because the effect of DT was significant $(P$ value $<0.05)$ on all responses, multiple means comparison was completed using Duncan's multiple range test at the $5 \%$ level of significance, and letter groupings were generated. The analysis was completed using the GLM Procedure of SAS (SAS Institute Inc., 2010).

Regression analysis to model the relationship between DT and essential oil content as well as the concentration of each of the constituents suggested that six of them (essential oil content and the concentration of linalool, methyl chavicol, transanethole, epoxy-pseudoisoeugenyl-2-methyl, and transpseudoisoeugenyl-2-methyl) can be adequately modeled (Bates and Watts, 2007) using the Asymptotic (Eq. 1), Power (Eq. 2), or the third-order polynomial (Eq. 3) model. There was no clear relationship between DT and the concentration of para-anis-aldehyde and gamma-himachalene. The relationship between DT and the yields of all seven constituents was adequately modeled by either the Asymptotic (Eq. 1) or the third-order polynomial (Eq. 3) model. Although the Asymptotic and the Power models are nonlinear, the third-order polynomial model is linear. The parameters of the nonlinear models were estimated iteratively using the NLIN Procedure of SAS (SAS Institute Inc., 2010).

$$
\begin{aligned}
& Y=\theta_{1}-\theta_{2} e^{-\theta_{3} x}+\varepsilon \\
& Y=\theta_{1} x^{\theta_{2}}+\varepsilon \\
& Y=\beta_{0}+\beta_{1} x+\beta_{2} x^{2}+\beta_{3} x^{3}+\varepsilon
\end{aligned}
$$

where $Y$ is the dependent (response) variable, $x$ is the independent (DT) variable, and the error term $\epsilon$ is assumed to have normal distribution with constant variance.

\section{Results}

Increasing DT from 5 to $360 \mathrm{~min}$ increased essential oil yield from anise seed from $0.09 \mathrm{~g} / 100 \mathrm{~g}$ to $2.01 \mathrm{~g} / 100 \mathrm{~g}$, respectively (Table 1). Further increase in DT to 480 min did not significantly increase essential oil yields.

The concentrations of linalool $(0.05 \%$ to $0.237 \%$ range) and methyl chavicol $(0.7 \%$ to $1.38 \%$ range) in the essential oil were highest at 5-min DT (the shortest DT) and gradually decreased with increasing DT (Table 1; Fig. 1). However, the yield of these two constituents increased with increasing DT to reach maximum at $120 \mathrm{~min}$ and 360 to $480 \mathrm{~min}$ for linalool and methyl chavicol, respectively (Table 2; Fig. 2). As expected, these constituents, with lower boiling points relative to the others, are eluted first so that their concentration in the early distillate is high and is diluted by other constituents at the long DTs.

The concentration of para-anis-aldehyde ( $0.38 \%$ to $0.75 \%$ range in the essential oil) was generally highest at 30-min DT and lowest at 360- to 480-min DT (Table 1). However, the yield of this constituent increased with increasing DT to reach a maximum at $120 \mathrm{~min}$ (Table 2), indicating the constituent continued to be eluted until $120 \mathrm{~min}$ and did not change significantly after that. The concentration of transanethole, the major anise oil constituent, varied within a relatively narrow range (from $93.5 \%$ to $96.2 \%$ in the essential oil); however, it was affected significantly by DT. Generally, the concentration of transanethole was highest at 15- to 60-min DT, low at 240 to $360 \mathrm{~min}$, and the lowest at 480-min DT (Table 1; Fig. 1). However, the yield of transanethole increased with increasing DT to reach maximum at 360 -min DT (Table 2; Fig. 2).

The concentration of para-anis-aldehyde ( $0.38 \%$ to $0.75 \%$ range in the essential oil) was highest at 30-min DT and lowest at 360min DT (Table 1). However, the yield of para-anis-aldehyde increased to reach maximum at 120-min DT (Table 2). Further increase in DT did not affect the yield of this oil constituent. The concentration of gamma-himachalene ( $0.58 \%$ to $1.25 \%$ range) was higher at 5-min and 480-min DT and the lowest at 180-min DT (Table 1), whereas the yield of this constituent increases steadily with increasing DT to reach maximum at 480-min DT (Table 2; Fig. 2). Both the concentrations and the yields of transpseudoisoeugenyl-2methyl $(0.03 \%$ to $1.53 \%$ range $)$ and epoxypseudoisoeugenyl-2-methyl $(0.013 \%$ to $0.563 \%$ range in the essential oil) increased with increasing DT to reach maximum values at 360-min or 480-min DT (Tables 1 and 2; Figs. 1 and 2).

The regression analyses results show that the Asymptotic model (Eq. 1), which is nonlinear, can be used to predict essential oil content and the yields of linalool, methyl chavicol, para-anis-aldehyde, and transanethole at any given DT using the fitted models in Figs. 1 and 2. On the other hand, the concentrations of linalool, methyl chavicol, transpseudoisoeugenyl-2-methyl, and

\begin{tabular}{|c|c|c|c|c|c|c|c|c|}
\hline & $\begin{array}{c}\text { EO } \\
\text { content }\end{array}$ & Linalool & $\begin{array}{c}\text { Methyl } \\
\text { chavicol }\end{array}$ & $\begin{array}{l}\text { Para-anis- } \\
\text { aldehyde }\end{array}$ & $\begin{array}{c}\text { Trans- } \\
\text { anethole }\end{array}$ & $\begin{array}{c}\text { Gamma- } \\
\text { himachalene }\end{array}$ & $\begin{array}{c}\text { Trans- } \\
\text { pseudoisoeugenyl- } \\
\text { 2-methyl }\end{array}$ & $\begin{array}{c}\text { Epoxy- } \\
\text { pseudoisoeugenyl- } \\
\text { 2-methyl }\end{array}$ \\
\hline DT $(\min )$ & \multicolumn{8}{|c|}{ (\%) of total oil } \\
\hline 5 & $0.09 \mathrm{~g}$ & $0.237 \mathrm{a}$ & $1.38 \mathrm{a}$ & $0.570 \mathrm{abc}$ & $95.5 \mathrm{abc}$ & $1.09 \mathrm{a}$ & $0.03 \mathrm{~d}$ & $0.013 \mathrm{e}$ \\
\hline 30 & $0.54 \mathrm{ef}$ & $0.103 \mathrm{bc}$ & $1.04 \mathrm{~b}$ & $0.747 \mathrm{a}$ & $96.2 \mathrm{a}$ & $0.66 \mathrm{bc}$ & $0.13 \mathrm{~d}$ & $0.073 \mathrm{e}$ \\
\hline 60 & $0.78 \mathrm{e}$ & $0.087 \mathrm{~cd}$ & $0.99 \mathrm{bc}$ & $0.657 \mathrm{ab}$ & $96.2 \mathrm{a}$ & $0.67 b c$ & $0.22 \mathrm{~d}$ & $0.117 \mathrm{de}$ \\
\hline 120 & $1.20 \mathrm{~d}$ & $0.083 \mathrm{cde}$ & $0.93 \mathrm{bc}$ & $0.667 \mathrm{ab}$ & $95.6 \mathrm{ab}$ & $0.67 \mathrm{bc}$ & $0.53 \mathrm{c}$ & $0.257 \mathrm{~cd}$ \\
\hline 360 & $2.01 \mathrm{a}$ & $0.050 \mathrm{e}$ & $0.70 \mathrm{~d}$ & $0.380 \mathrm{c}$ & $94.8 \mathrm{c}$ & $0.83 \mathrm{~b}$ & $1.27 \mathrm{ab}$ & $0.447 \mathrm{ab}$ \\
\hline 480 & $1.86 \mathrm{ab}$ & $0.067 \mathrm{de}$ & $0.79 \mathrm{~cd}$ & $0.520 \mathrm{bc}$ & $93.5 \mathrm{~d}$ & $1.25 \mathrm{a}$ & $1.53 \mathrm{a}$ & $0.563 \mathrm{a}$ \\
\hline
\end{tabular}

Table 1. Mean essential oil (EO) content (\%) and the concentrations (\%) of linalool, methyl chavicol, para-anis-aldehyde, transanethole, gamma-himachalene, transpseudoisoeugenyl-2-methyl, and epoxy-pseudoisoeugenyl-2-methyl obtained from the nine distillation times (DTs). ${ }^{z}$

${ }^{\mathrm{z}}$ Within each column, means sharing the same letter are not significantly different at the $5 \%$ level. 

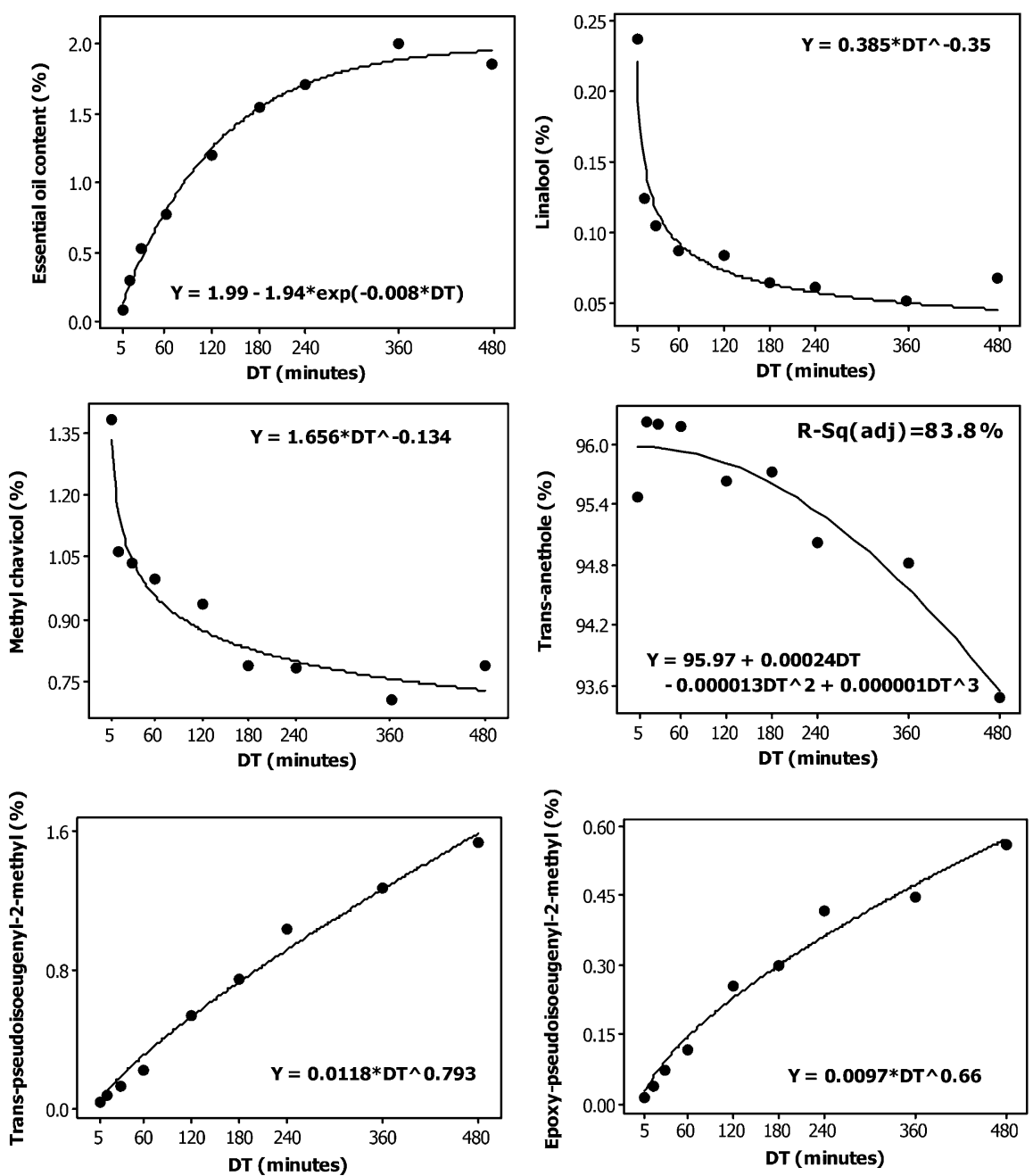

Fig. 1. Plot of essential oil content and the concentration of five constituents vs. distillation time (DT) along with the fitted Asymptotic, Power, or third-order polynomial regression models. Equations of the fitted models are shown within each plot. The adjusted coefficient of determination $\left[R^{2}(\operatorname{adj})\right]$ is given for the linear (third-order polynomial) model only.

Table 2. Mean yield (mg) of linalool, methyl chavicol, para-anis-aldehyde, transanethole, gammahimachalene, transpseudoisoeugenyl-2-methyl, and epoxy-pseudoisoeugenyl-2-methyl obtained from the nine distillation times (DTs). ${ }^{2}$

\begin{tabular}{|c|c|c|c|c|c|c|c|}
\hline \multirow{2}{*}{$\begin{array}{l}\text { DT } \\
(\min )\end{array}$} & Linalool & $\begin{array}{l}\text { Methyl } \\
\text { chavicol }\end{array}$ & $\begin{array}{c}\text { Para- } \\
\text { anis- } \\
\text { aldehyde }\end{array}$ & $\begin{array}{l}\text { Trans- } \\
\text { anethole }\end{array}$ & $\begin{array}{c}\text { Gamma- } \\
\text { himachalene }\end{array}$ & $\begin{array}{c}\text { Trans- } \\
\text { pseudoisoeugenyl- } \\
\text { 2-methyl }\end{array}$ & $\begin{array}{c}\text { Epoxy- } \\
\text { pseudoisoeugenyl- } \\
\text { 2-methyl }\end{array}$ \\
\hline & \multicolumn{7}{|c|}{ (mg per 100 g seed) } \\
\hline 5 & $0.211 \mathrm{e}$ & $1.24 \mathrm{~h}$ & $0.52 \mathrm{c}$ & $87 \mathrm{f}$ & $0.98 \mathrm{~h}$ & $0.03 \mathrm{e}$ & $0.01 \mathrm{e}$ \\
\hline 15 & $0.361 \mathrm{de}$ & $3.12 \mathrm{~g}$ & $1.92 \mathrm{c}$ & $285 \mathrm{ef}$ & $2.27 \mathrm{~g}$ & $0.22 \mathrm{e}$ & $0.12 \mathrm{e}$ \\
\hline 30 & $0.552 \mathrm{~cd}$ & $5.54 \mathrm{f}$ & $4.02 \mathrm{~b}$ & $515 \mathrm{de}$ & $3.54 \mathrm{f}$ & $0.68 \mathrm{de}$ & $0.40 \mathrm{e}$ \\
\hline 60 & $0.671 \mathrm{bc}$ & $7.71 \mathrm{e}$ & $5.14 \mathrm{~b}$ & $748 \mathrm{~d}$ & $5.12 \mathrm{e}$ & $1.71 \mathrm{~d}$ & $0.92 \mathrm{e}$ \\
\hline 120 & $1.001 \mathrm{a}$ & $11.21 \mathrm{~d}$ & $8.03 \mathrm{a}$ & $1150 \mathrm{c}$ & $8.08 \mathrm{~d}$ & $6.36 \mathrm{c}$ & $3.10 \mathrm{de}$ \\
\hline 180 & $0.950 \mathrm{ab}$ & $11.93 \mathrm{~cd}$ & $8.36 \mathrm{a}$ & $1476 \mathrm{~b}$ & $8.89 \mathrm{~d}$ & $11.39 \mathrm{~b}$ & $4.66 \mathrm{~cd}$ \\
\hline 240 & $1.013 \mathrm{a}$ & $13.13 \mathrm{bc}$ & $9.09 \mathrm{a}$ & $1624 \mathrm{~b}$ & $11.99 \mathrm{c}$ & $17.62 \mathrm{~b}$ & $7.15 \mathrm{bc}$ \\
\hline 360 & $1.000 \mathrm{a}$ & $14.11 \mathrm{ab}$ & $7.62 \mathrm{a}$ & $1904 \mathrm{a}$ & $16.70 \mathrm{~b}$ & $25.40 \mathrm{a}$ & $8.95 \mathrm{ab}$ \\
\hline 480 & $1.236 \mathrm{a}$ & $14.61 \mathrm{a}$ & $9.64 \mathrm{a}$ & $1741 \mathrm{ab}$ & $23.19 \mathrm{a}$ & $28.40 \mathrm{a}$ & $10.52 \mathrm{a}$ \\
\hline
\end{tabular}

${ }^{\mathrm{z}}$ Within each column, means sharing the same letter are not significantly different at the $5 \%$ level.

epoxy-pseudoisoeugenyl-2-methyl were described well by the nonlinear Power model (Eq. 2), and the fitted models shown in Fig. 1 can be used to predict these concentrations at any DT. However, there was no nonlinear regression model that describes the relationship between DT and the concentration of transanethole and the yields of gammahimachalene, transpseudoisoeugenyl-2-methyl, and epoxy-pseudoisoeugenyl-2-methyl. The third-order polynomial model (fitted models shown in Figs. 1 and 2) was the best available model to describe the relationships.

\section{Discussion}

Anise essential oil yield and composition can be altered by growing conditions and genotype (Leela and Vipin, 2008; Ullah and Honermeier, 2012) and by the type of extraction (Ondarza and Sánchez, 1990). A previous study (Balinova-Tsvetkova and Kamburova, 1975) reported that $82 \%$ of the essential oil of whole anise seed was extracted during the first hour of distillation; however, it took $4 \mathrm{~h}$ to extract all of the oil and to complete the distillation. The use of a range of DT allowed us to build regression models that can be used to predict essential oil yield, the concentration of individual constituents, and the yield of these constituents with DT starting at $5 \mathrm{~min}$ and finishing with $480 \mathrm{~min}$

In some instances, the relative concentration of some constituents in the oil decreased with increase of the DT; however, their yield increased with an increase of the DT. This was the result of the increase in the essential oil yield (the yield of any constituent was a function of its relative concentration in the oil and the essential oil yield). Apparently, some of the constituents were eluted first and their concentration in the oil from shorter DT was high. However, because other constituents were eluted with longer DT, the concentration of the constituents eluted first in the oil would be decreased as a result of the dilution effect. For example, in this study, the concentration of transanethole decreased at 360 and then decreased further at 480-min DT relative to the shorter DT. However, the yield of transanethole at 360 and at 480-min DT was greater than its yield at shorter DT. This demonstrates that transanethole continued to be eluted up to 360 -min DT. The yield of transanethole at 480-min DT was not different from its yield at 360-min DT, indicating that either 1) no more transanethole was eluted after $360 \mathrm{~min}$; or 2) part of transanethole could have been converted into another constituent between 360 and 480-min DT. Data from this study are not sufficient to ascertain the latter possibility; this can be verified with further studies. The observed differential trends between the concentration and yield of a given constituent of the essential oil has been observed in DT with other crops (e.g., for menthone and other constituents of Mentha canadensis in Zheljazkov and Astatkie, 2012; for alfa-pinene, sabinene, myrcene, and other constituents of Juniperus scopulorum in Zheljazkov et al., 2012c).

The study demonstrated the need for reporting DT for anise seed when anise essential oil yield and composition are discussed because DT can significantly affect the essential oil yield and composition. Also, this article can be used as a reference point for comparing studies in which different DTs were used for anise seed essential oil. Moreover, the results can be readily used by the 

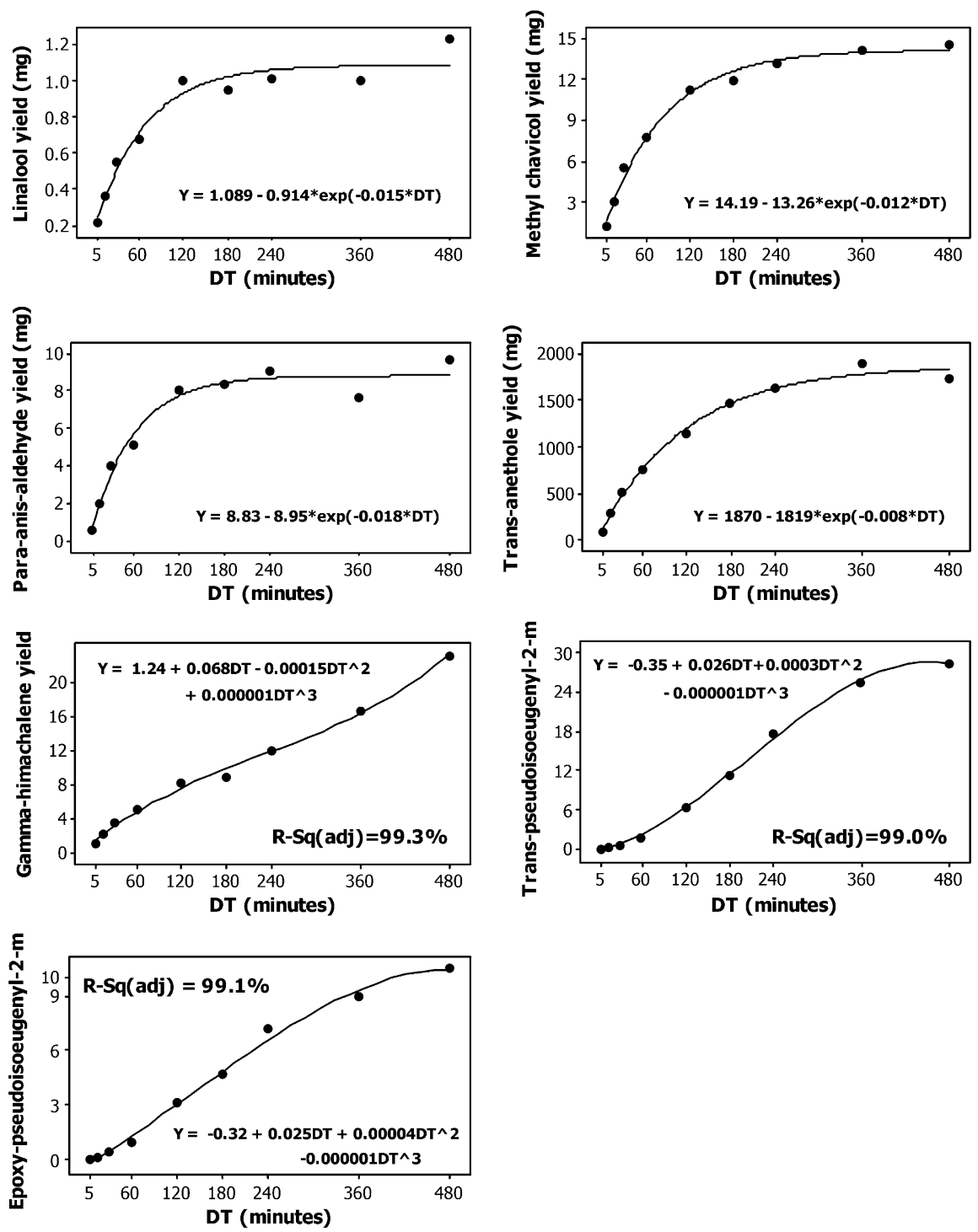

Fig. 2. Plot of the yield (mg) of seven constituents vs. distillation time along with the fitted Asymptotic or third-order polynomial regression models. Equations of the fitted models are shown within each plot. The adjusted coefficient of determination $\left[R^{2}(\operatorname{adj})\right]$ is given for the linear (third-order polynomial) model only.

essential oil industry. Anise seed should be steam-distilled for $360 \mathrm{~min}$ to obtain the highest essential oil yield and to obtain a maximum yield of transanethole, the major oil constituent.

\section{Literature Cited}

Balinova-Tsvetkova, A. and K. Kamburova. 1975. Determination of the essential oil content in the seed of some Umbelliferae. Rastenievadni Nauki. 12:40-45.

Bates, D.M. and D.G. Watts. 2007. Nonlinear regression and its applications. Wiley, New York, NY

Bluma, R.V. and M.G. Etcheverry. 2008. ApplicaAspergillus section Flavi growth parameters and aflatoxin accumulation. Food Microbiol. 25:324-334. tion of essential oils in maize grain: Impact on
Cannon, J.B., C.L. Cantrell, T. Astatkie, and V.D. Zheljazkov. 2013. Modification of yield and composition of essential oils by distillation time. Ind. Crops Prod. 41:214-220.

Gawde, A.J., C.L. Cantrell, and V.D. Zheljazkov. 2009. Dual extraction of essential oil and podophyllotoxin from Juniperus virginiana. Ind. Crops Prod. 30:276-280.

Gende, L.B., M.D. Maggi, R. Fritz, M.J. Eguaras, P.N. Bailac, and M.I. Ponzi. 2009. Antimicrobial activity of Pimpinella anisum and Foeniculum vulgare essential oils against Paenibacillus larvae. J. Essent. Oil Res. 21: 91-93.

Leela, N.K. and T.M. Vipin. 2008. Aniseed, p. 331341. In: Parthasarathy, V.A., B. Chempakam, and T.J. Aachariah (eds.). Chemistry of spices. CABI, Wallingford, UK.

Montgomery, D.C. 2013. Design and analysis of experiments. 8th Ed. Wiley, New York, NY.

Ondarza, M. and A. Sánchez. 1990. Steam distillation and supercritical fluid extraction of some Mexican spices. Chromatographia 30:16-18.

SAS Institute Inc. 2010. SAS/STAT ${ }^{\circledR} 9.3$ user's guide. SAS Institute Inc., Cary, NC.

Stojanov, N. 1973. Anise, Pimpinella anisum, p. 220-224. In: Stojanov, N. (ed.) Our medicinal plants. Nauka I Izkustvo Press, Sofia, Bulgaria.

Tonutti, I. and P. Liddle. 2010. Aromatic plants in alcoholic beverages. A review. Flavour Fragrance J. 25:341-350.

Ullah, H. and B. Honermeier. 2012. Fruit yield, essential oil concentration and composition of three anise cultivars (Pimpinella anisum L.) in relation to sowing date, sowing rate and locations. Ind. Crops Prod. 42:489-499.

Zheljazkov, V.D. and T. Astatkie. 2012. Effect of distillation time on Mentha canadensis essential oil yield and composition. HortScience 47: 643-647.

Zheljazkov, V.D., T. Astatkie, and V. Schlegel. 2012a. Effects of distillation time on the Pinus ponderosa essential oil yield, composition, and antioxidant activity. HortScience 47:785-789.

Zheljazkov, V.D., T. Astatkie, and V. Schlegel. 2012b. Distillation time changes oregano essential oil yields and composition but not the antioxidant or antimicrobial activities. HortScience 47:777-784.

Zheljazkov, V.D., T. Astatkie, E.A. Jeliazkova, and V. Schlegel. 2012c. Distillation time alters essential oil yield, composition, and antioxidant activity of male Juniperus scopulorum trees. J. Oleo Sci. 61:537-546.

Zheljazkov, V.D., T. Astatkie, E.A. Jeliazkova, A.O. Tatman, and V. Schlegel. 2013. Distillation time alters essential oil yield, composition and antioxidant activity of female Juniperus scopulorum trees. J. Essent. Oil Res. 25:62-69.

Zheljazkov, V.D., C.L. Cantrell, B. Tekwani, and S. Khan. 2008. Content, composition, and bioactivity of the essential oil of three basil genotypes as a function of harvesting. J. Agr. Food Chem. 56:380-385. 